\title{
Electron Beam Generation in Tevatron Electron Lenses
}

\author{
V.Kamerdzhiev, G.Kuznetsov, V.Shiltsev ${ }^{1}$, N.Solyak \\ Fermi National Accelerator Laboratory, PO Box 500, Batavia, IL 60510, USA
}

M.Tiunov

Budker Institute of Nuclear Physics, Novosibirsk, 630090, Russia

\begin{abstract}
New type of high perveance electron guns with convex cathode has been developed. Three guns described in this article are built to provide transverse electron current density distributions needed for Electron Lenses for beam-beam compensation in the Tevatron collider. The current distribution can be controlled either by the gun geometry or by voltage on a special control electrode located near cathode. We present the designs of the guns and report results of beam measurements on the test bench. Because of their high current density and low transverse temperature of electrons, electron guns of this type can be used in electron cooling and beambeam compensation devices.
\end{abstract}

Keywords: electron gun, high perveance, proton-antiproton collider

PACS: 29.25.Bx, 41.85.Ct, 41.85.Ew, 41.85.Qg

\section{INTRODUCTION: ELECTRON BEAM FOR BEAM-BEAM COMPENSATION}

Fermilab's Tevatron is currently the worlds' highest energy proton antiproton collider operating at $980 \mathrm{GeV}$ energy per beam. In the Collider Run II (2001-present) it operates with six times more bunches, many times higher beam intensities and luminosities than in Run I (1992-1995). Electromagnetic long-range and head-on interactions of high intensity proton and antiproton beams have been significant sources of beam loss and lifetime limitations [1]. One of the possibilities to mitigate these effects is to employ so called "electron lenses" as proposed in [2]. In such a scheme, beam of negatively charged low-energy electrons collides with negatively charged high-energy antiprotons and, thus, acting as a defocusing lens (as electrons repel antiprotons) which compensates focusing effects due to collision with highcurrent proton beam at the main interaction points.

The figure of merit of the interaction is shift of the antiproton tune $v=f_{\beta} / f_{0}$ which is ratio of the antiproton betatron oscillation frequency $f_{a}$ to the revolution frequency $f_{0}$. The betatron tune shift due to electrons is proportional to the electron density $n_{e}$ :

$$
\Delta v_{x, y}=-n_{e} \frac{\beta_{x, y} L r_{\bar{p}}}{2 \gamma_{\bar{p}}}
$$

\footnotetext{
${ }^{1}$ shiltsev@fnal.gov
} 
here $\beta_{x, y}$ is horizontal/vertical beta-function at the location of the electron lens, $L$ is electron beam length, $r_{\bar{p}}=1.53 \cdot 10^{-15} \mathrm{~m}$ is classical (anti)proton radius and $\gamma_{\bar{p}}$ is relativistic factor of antiprotons. To shift the tune of $980 \mathrm{GeV}$ beam by $\Delta v \approx-0.01$, one needs a 2 meter long electron lens at the location with $\beta_{x, y}=100 \mathrm{~m}$ with $n_{e} \approx 5 \cdot 10^{10} \mathrm{~cm}^{-3}$. The latter is equivalent to the current density of $j_{e}=e n_{e} v_{e} \approx 50 \mathrm{~A} /$ $\mathrm{cm}^{2}$ of $10 \mathrm{kV}$ electrons.

Two Tevatron electron lenses (TEL) have been built and installed in the Tevatron, one of them is in routine operation since 2001, another one is being commissioned. Layout of the TEL is shown in Fig.1. Electron beam in TELs is strongly magnetized all the way from cathode to the interaction region to the electron collector [3]. Magnetic system of TEL allows magnetic compression of the electron beam cross section area by factor of $\sim 10$, so the required maximum electron current density at the cathode is about $j_{c}=j_{e} / 10 \approx 5 \mathrm{~A} / \mathrm{cm}^{2}$. The cathode radius should be $0.5-1 \mathrm{~cm}$ Because of significant differences in dynamics of different bunches, the electron current has to be modulated with high duty factor and characteristic on-off time of about 0.5-1 microsecond. High current density, fast modulation and requirement of smooth current density profile led to choice of the electron gun with convex cathode (to get higher perveance - see below) and modulation by anode voltage (no grid). During experimental beam studies in Tevatron, several electron current profiles were found out to be effective: a) rectangular distribution results in uniform tune shift $\Delta v$ for all high energy particles inside electron beam, but has disadvantage of strong nonlinear space charge forces beyond boundaries of electron beam; b) bell-shape (close to Gaussian) has smaller nonlinearities but smaller beam size as well, that makes it somewhat cumbersome to align it on the beam of (anti)protons; c) "smoothed-edge-with-flat-top" (SEFT) distribution combines advantages of both previously mentioned distributions.

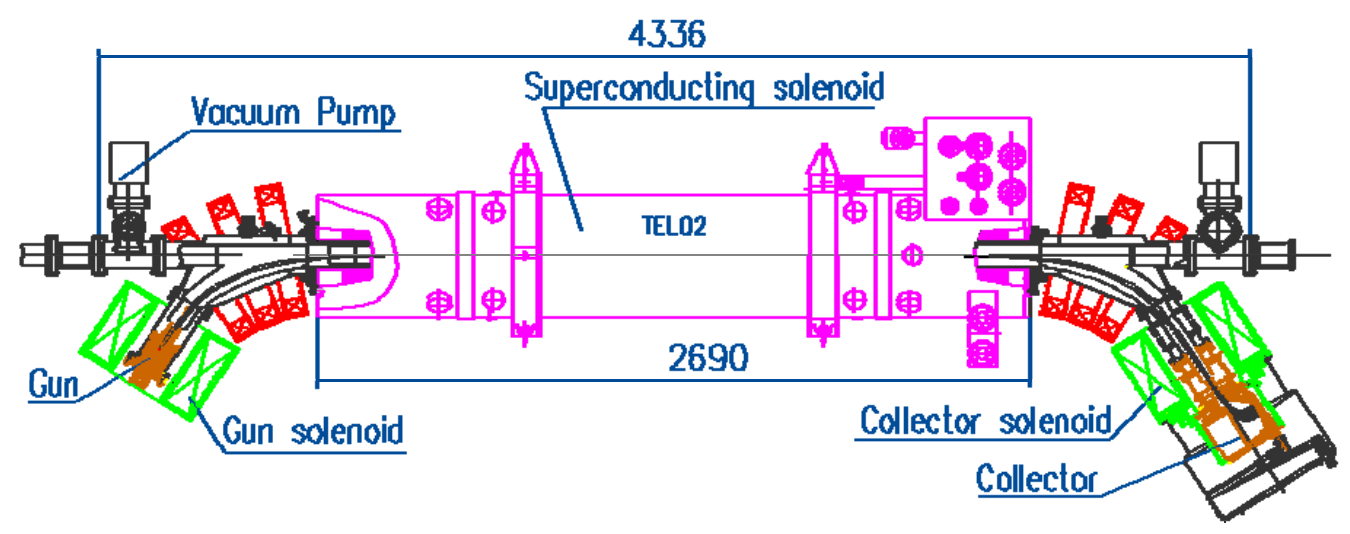

FIGURE 1. Layout of the Tevatron Electron Lens.

We have developed three electron guns to satisfy all the above mentioned needs of the Tevatron electron lenses for beam-beam compensation. One of the most important characteristics of an electron gun is its perveance $P=I / U_{a}^{3 / 2}$, where $I$ is the beam 
current and $U_{a}$ is the anode potential with respect to the cathode. For guns with flat or concave cathodes, current density inhomogeneity becomes large when the perveance exceeds the value of $1-2 \mu \mathrm{A} / \mathrm{V}^{3 / 2}$. For these and similar cases, where the gun has to be immersed into a strong longitudinal magnetic field, the perveance can be increased by usage of a convex cathode [4]. All the guns were tested at the "Tevatron Electron Lens" (TEL) prototype set-up at Fermilab [5]. The paper presents results of the gun designs and test measurements.

\section{GUN DESIGN AND TEST SETUP}

The electron guns were simulated and optimised using UltraSAM code [6] in order to have desired current density distribution and high perveance. The geometries of the guns are shown in Fig.2 together with electric field distribution along the beam axis (the guns have axial symmetry) and electron trajectories. The guns employ spherical cathodes with +-45 deg opening angle. A "control electrode" around the cathode (an analog of the Pierce electrode) is employed in the "flat" gun (Fug.2a) for manipulation of the beam current density distribution. Electrodes with somewhat different geometry are installed for the same purpose between cathode and anode in the "Gaussian" and SEFT guns (Figs.2b and c). Near cathode electrodes in those two guns are always at cathode potential. To get desired current distribution, voltage on the "control" electrodes - controlled by a separate power supply - should be the same as cathode voltage.

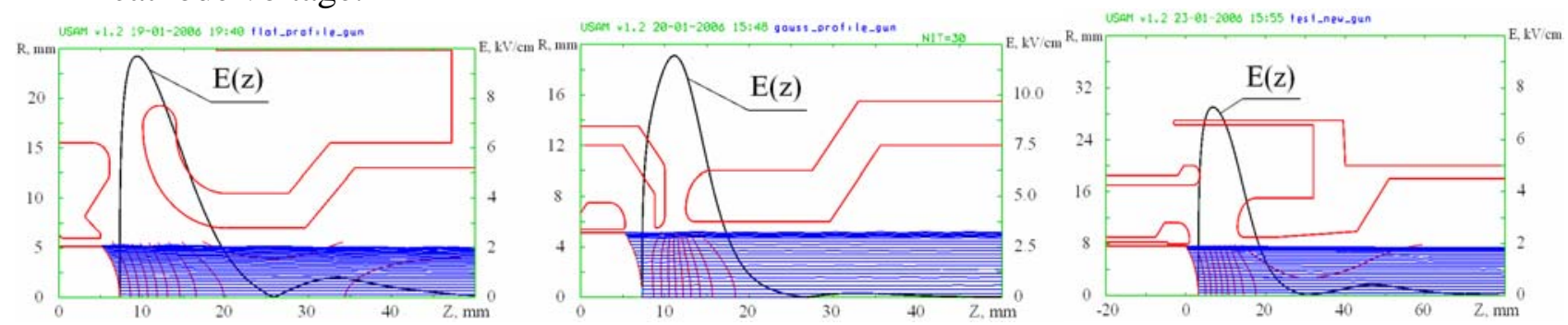

FIGURE 2. UltraSAM code geometry and electric field simulation results for: a) (left) "flat" gun; b) (center) Gaussian gun; c) (right) SEFT gun.

Mechanically, all three guns look similar - see Fig.3a). They are assembled on $63 / 4$ " SS vacuum flange and use ceramic rings as insulators between electrodes. The guns employ spherical convex dispenser cathodes made by HeatWave Labs (Watsonville, CA). 10 and $15 \mathrm{~mm}$ diameter Barium impregnated Tungsten cathodes operate at temperatures $950-1200^{\circ} \mathrm{C}$. They are equipped with MoRe support sleeve and Molybdenum mounting flange and have internal heater filament (bifilar option, one heater lead internally grounded). Near cathode electrodes are made of Molybdenum, control electrodes and anodes are made of oxygen free Copper.

Gun characteristics were measured on a test bench used at Fermilab for prototyping of TEL elements [5]. The test bench consists of the gun immersed into longitudinal magnetic field $B_{\text {gun }}$ of 1- $2 \mathrm{kG}$ generated by a gun solenoid, a drift tube with diagnostics placed inside $4 \mathrm{kG}, 2 \mathrm{~m}$ long main solenoid, and a collector, also inside a separate solenoid. The collector is equipped with a beam analyzer - see Fig. 
3b). A small ( $\varnothing 0.2 \mathrm{~mm}$ ) hole in the collector bottom cuts a narrow part of the electron beam, which passes through a retarding electrode and is absorbed by an analyzer collector. To measure the current density distribution, the beam is moved with respect the hole by steering coils placed inside the main solenoid, and the analyzer collector current $I_{a c}$ is recorded as a function of the beam transverse position

If the potential of the retarding electrode with respect to the cathode $U_{r}$ is close to zero, only electrons having high enough longitudinal momentum $P_{\|}$reach the analyzer collector. Derivation of the measured function $I_{a c}\left(U_{r}\right)$ gives an electron distribution over "longitudinal energy" $E_{\|}=\frac{P_{\|}^{2}}{2 m}-e U_{r}$.
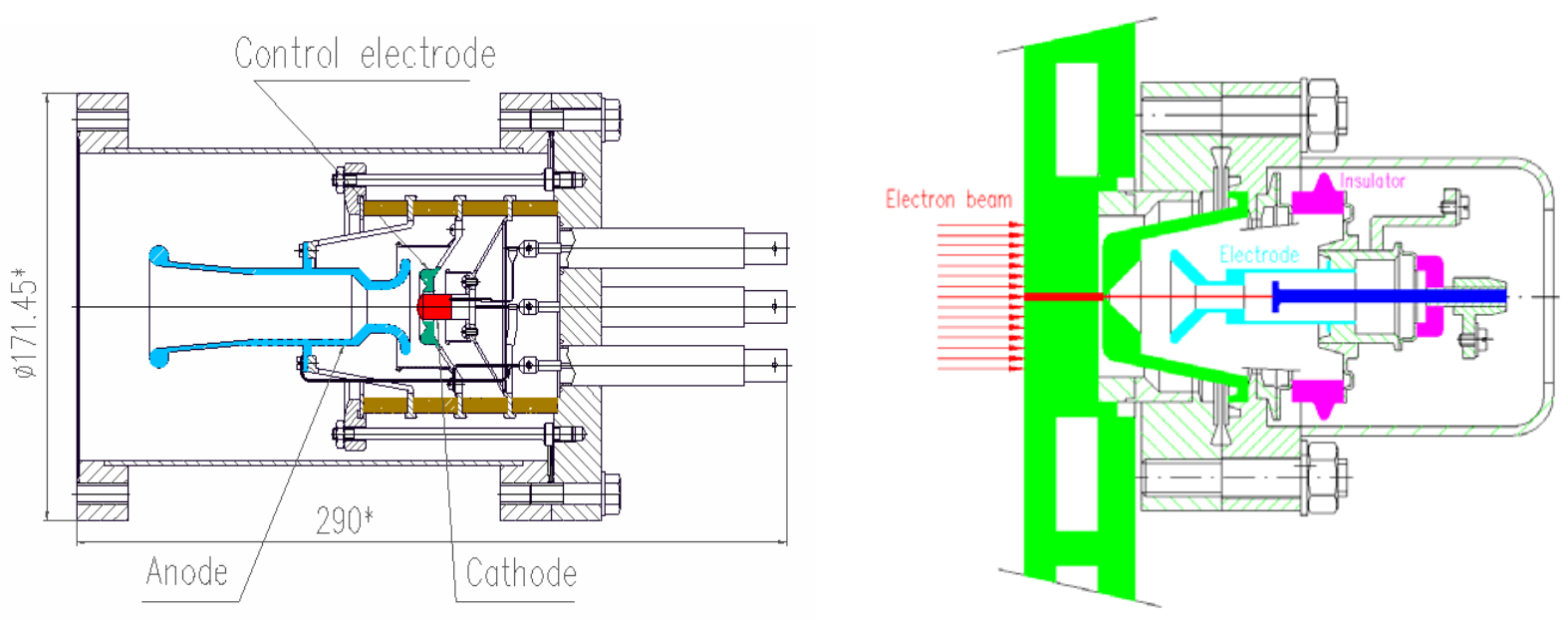

FIGURE 3. a) (left) Mechanical design of the "flat" gun; b) (right) pin-hole collector assembly for beam profile measurements.

\section{RESULTS OF MEASUREMENTS}

Except high perveance, the guns are not much different from a planar cathode gun. The beam currents follow the Child's law with a good precision (Fig.4a) yielding perveances of $5.3 \mu \mathrm{A} / \mathrm{V}^{3 / 2}, 4.3 \mu \mathrm{A} / \mathrm{V}^{3 / 2}, 1.8 \mu \mathrm{A} / \mathrm{V}^{3 / 2}$ for "flat", SEFT and "Gaussian" guns, respectively. To prevent thermal problems at the irradiated surface, total current, profile and temperature measurements were done in the DC regime at currents below 0.5-1 A. Gun characteristics at higher currents was investigated in a pulsed regime with the pulse width of $0.2-1 \mu \mathrm{s}$. No significant deviation from results of the DC measurements was found.

Measured distributions of the longitudinal energy of electrons are close to the Gaussian one:

$$
\frac{d I_{a c}}{d E_{\|}}=\frac{1}{\sqrt{2 \pi}} \cdot \frac{I_{a c 0}}{\delta W} \cdot \exp \left\{-\frac{\left(E_{\|}-E_{0}\right)^{2}}{2 \delta W^{2}}\right\},
$$

where $I_{a c 0}$ is the maximum value of the analyzer collector current typically measured at the potential of the retarding electrode equal to $100 \mathrm{~V}$ with respect to the cathode. The energy spread $\delta W$, measured in the beam center, depends on the beam current in a 
good accordance with known formulae, describing relaxation in the beam [7], in the entire range of measured beam currents (see Fig.4b). At currents below $0.2 \mathrm{~A}$, the measurements of $E_{0}$ were performed also near the beam periphery. The results were equal to ones obtained in the beam center within precision of measurements, which was about $1 \mathrm{~V}$. The fact implies that the possible "transverse energy" acquired in the gun is less than $1 \mathrm{eV}$.
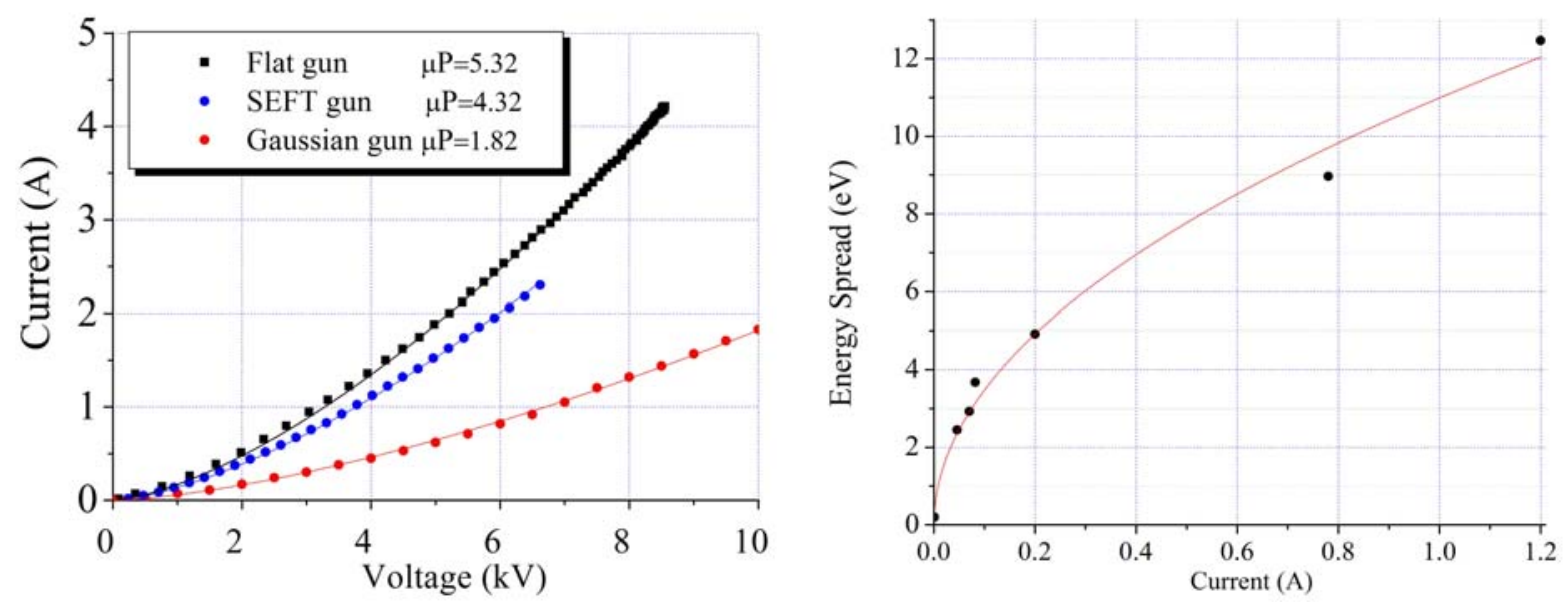

FIGURE 4. a) (left) Volt-Ampere characteristics of the three electron guns, solid lines are fits according to Child's law $P=I / U_{a}^{3 / 2}$; b) electron energy spread vs total beam current from "flat" gun. Magnetic field is $2 \mathrm{kG}$, solid line corresponds to the fit $\delta W[\mathrm{eV}]=10.8 \cdot \sqrt{I[A]}$.

Two dimensional profile of the electron current from the SEFT gun measured by the "pin-hole" collector is shown in Fig. 5a). Current density variations are less than $10 \%$ over $90 \%$ of beam diameter. Measured and calculated one dimensional profiles of electron beams from all three guns are presented in Fig. 5b). One can see very good agreement between predicted and observed current densities over most of beam area except the very edge of the beam.
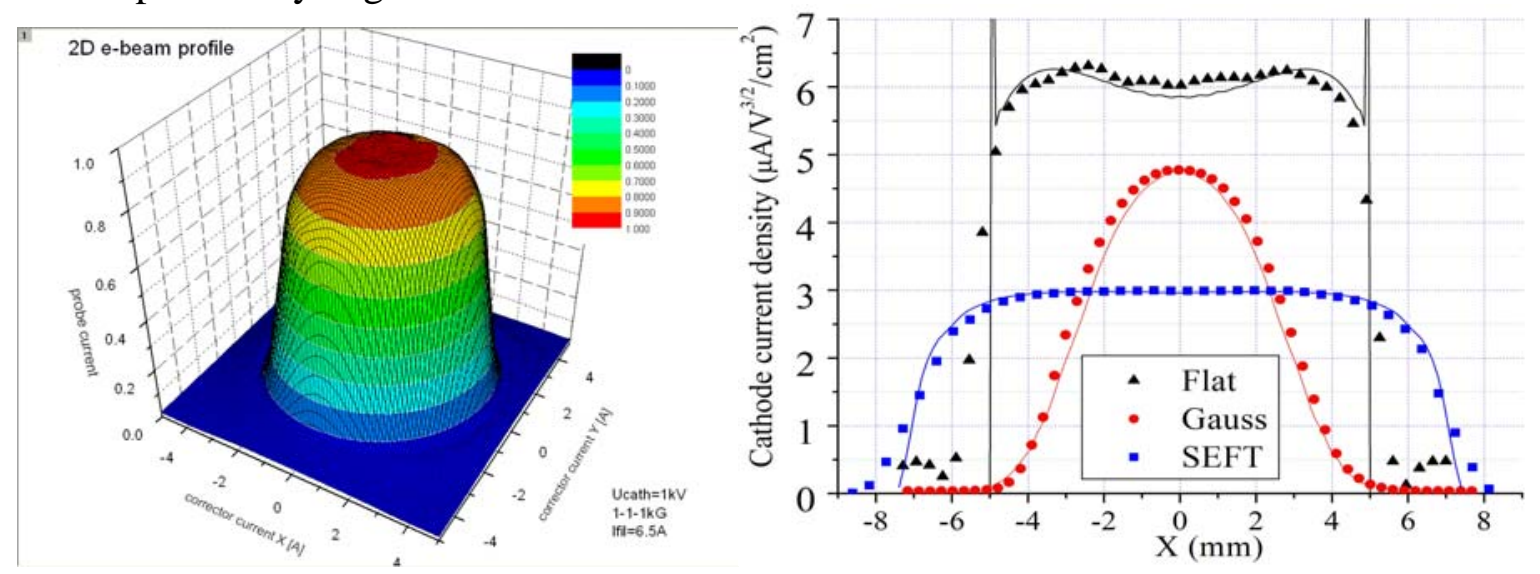

FIGURE 5. a) (left) 2D electron current density of SEFT gun beam; b) (right) 1D current density distributions for three guns, solid lines represent UltraSAM simulation results. In both cases, control electrode voltage was set equal to cathode voltage. 
Electron emission from the edges of the cathode is strongly dependent on accuracy of alignment of near cathode or control electrodes w.r.t. cathode. Fig. 6a) shows 1D current profile in the case when control electrode of the flat gun was (unintentionally) set a bit farther from the anode than the cathode. Edge peaks in the current density profile indicate, and computer simulations confirm, that the reason is some $0.4 \mathrm{~mm}$ protrusion of the emitted surface from the control electrode with respect to its optimum position. The shift occurs because of either uncertainty in the thermal expansion of the cathode or mechanical error. Probably, a slight current distribution asymmetry, seen in Fig.6a), is because of an asymmetric misalignment. Application of negative (w.r.t. cathode potential) voltages to the control electrode resulted in suppression of the edge emission and can lead to narrower almost bell-shape current profile. Total beam current reduction factors for SEFT and "flat" gun are shown on Fig.6b) as functions of (negative) control electrode voltage $U_{c e}$ normalized to (positive) anode-cathode voltage difference $U_{a c}$. Application of positive voltage to the control electrode results in formation of hollow beam which is disadvantageous for the beam-beam compensation purposes but can be used effectively for other applications, e.g., for electron cooling [8].
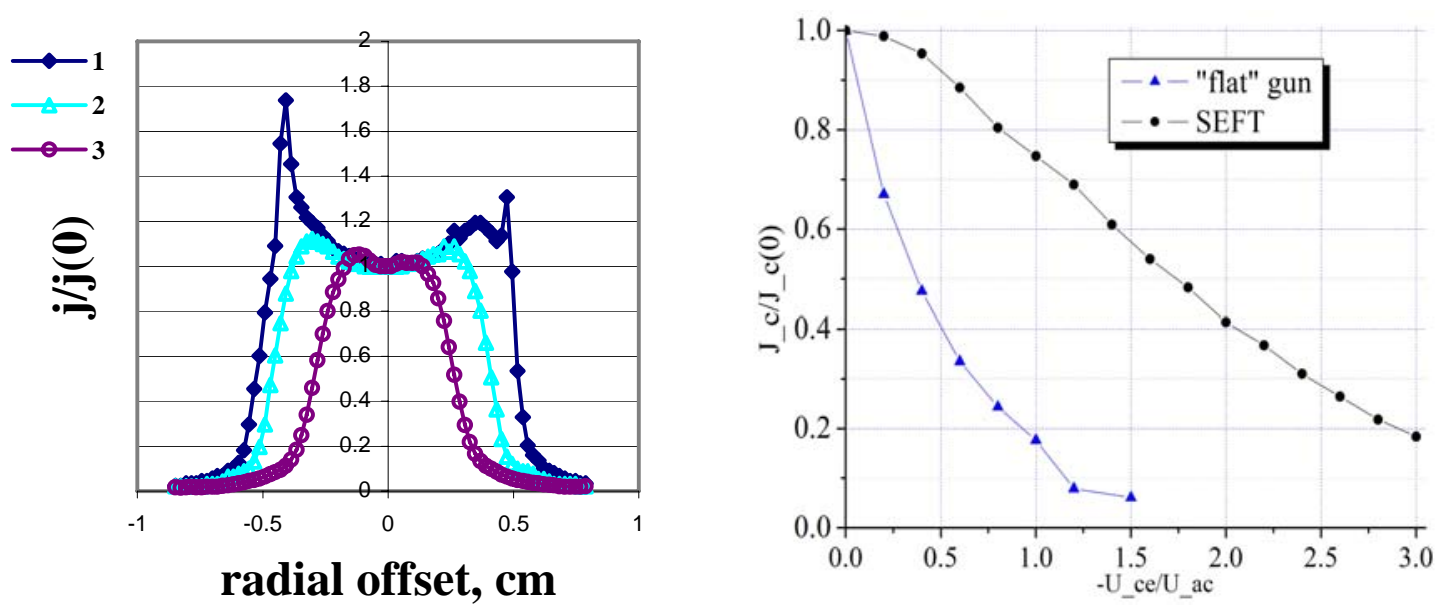

FIGURE 6. a) (left) Current density distributions for three control electrode voltages: $1-U_{c e}=0, I=1$ A; 2- $U_{c e}=-0.3 \mathrm{kV}, I=0.6 \mathrm{~A}, 3-U_{c e}=-1.2 \mathrm{kV}, I=0.16 \mathrm{~A}$. $U_{a}=3 \mathrm{kV}$, magnetic field in all solenoids $2 \mathrm{kG}$; b) (right) cathode current reduction in "flat" and SEFT guns vs control electrode voltage.

\section{CONCLUSION}

We have designed, built and tested three high-perveance thermionic electron guns with transverse electron current density distributions as specified by the needs of Beam-Beam Compensation experiment in the Tevatron collider. Results of gun measurements on a test stand satisfactorily agree with UltraSAM code simulations. High perveance of 1.8-5.3 $\mu \mathrm{A} / \mathrm{V}^{3 / 2}$ in the "flat", "Gaussian" and SEFT guns was due to use of convex cathodes. Control electrodes employed in the guns allow some variation of the current profiles. 


\section{ACKNOWLEDGMENTS}

Authors are thankful to A.Shemyakin for numerous inspiring discussions and to D.Wolff, H.Pfeffer, G.Saewert, B.Hively, T.Bolshakov, M.Kufer and J.Featherstone for help in commissioning and operations of the test set-up and both TELs.

TABLE 1: Main parameters of the tested guns

\begin{tabular}{|l|l|l|l|l|}
\hline Parameter & Gun \#1 & Gun \#2 & Gun \#3 & Units \\
\hline Cathode diameter & 10 & 10 & 15 & $\mathrm{~mm}$ \\
\hline Current profile & rectangular & Gaussian & SEFT & \\
\hline Gun perveance, max & 5.9 & 1.7 & 4.2 & $\mu \mathrm{A} / \mathrm{V}^{3 / 2}$ \\
\hline Max. current density & 6.3 & 4.8 & 3.0 & $\mu \mathrm{A} / \mathrm{V}^{3 / 2} / \mathrm{cm}^{2}$ \\
\hline Control voltage to shut off & 2.5 & 3 & 3.5 & $U_{\text {control }} / U_{\text {anode }}$ \\
\hline Filament power & $35-45$ & $35-45$ & $60-70$ & $\mathrm{~W}$ \\
\hline$B$-field on cathode & $1-4$ & $1-4$ & $1-4$ & $\mathrm{kG}$ \\
\hline
\end{tabular}

\section{REFERENCES}

1. V. Shiltsev, et al., "Beam-beam effects in Tevatron”, Phys. Rev. ST Accel. Beams 8, 101001 (2005).

2. V.Shiltsev, V.Danilov, D.Finley, A.Sery, "Considerations on compensation of beam-beam effects in the Tevatron with electron beams”, Phys. Rev. ST Accel. Beams 2, 071001 (1999).

3. N.Solyak, et al., "Electron Beam System of the Tevatron Electron Lens", in Proc. IEEE PAC 2001 (Chicago, IL, USA), p.1420.

4. A.Sharapa, A.Grudiev, D.Myakishev, A.Shemyakin, "A high-perveance electron gun for the electron cooling”, NIM- A406 (1998), p.169.

5. C.Crawford, et al, "Prototype Electron Lens setup for the Tevatron beam-beam compensation", in Proc. IEEE PAC 1999 (New-York,, NY, USA), p.237.

6. A. Ivanov, M. Tiunov, "ULTRASAM - 2D CODE FOR SIMULATION OF ELECTRON GUNS WITH ULTRA HIGH PRECISION”, Proceedings of EPAC 2002, Paris, France, pp.1634-1636.

7. N.Dikansky, et al, "Ultimate possibilities of electron cooling”, Preprint INP 88-61, Novosibirsk (1988).

8. A.Bubley, et al., "The electron gun with variable beam profile for optimization of electron cooling", in Proc. European PAC 2000 (Paris, France), p.1356. 